\title{
Molecular Imaging of Human Embryonic Stem Cells Stably Expressing Human PET Reporter Genes After Zinc Finger Nuclease-Mediated Genome Editing
}

\author{
Esther Wolfs*1, Bryan Holvoet*1, Laura Ordovas ${ }^{2,3}$, Natacha Breuls ${ }^{1,4}$, Nicky Helsen ${ }^{2,3}$, Matthias Schönberger ${ }^{5}$, \\ Susanna Raitano ${ }^{2,3}$, Tom Struys ${ }^{6}$, Bert Vanbilloen ${ }^{1}$, Cindy Casteels ${ }^{1}$, Maurilio Sampaolesi ${ }^{4}$, Koen Van Laere ${ }^{1}$, \\ Ivo Lambrichts ${ }^{6}$, Catherine M. Verfaillie ${ }^{\dagger 2,3}$, and Christophe M. Deroose ${ }^{\dagger 1}$ \\ ${ }^{I}$ Molecular Small Animal Imaging Centre, Nuclear Medicine and Molecular Imaging, Department of Imaging and Pathology, KU \\ Leuven, Leuven, Belgium; ${ }^{2}$ Stem Cell Institute, KU Leuven, Leuven, Belgium; ${ }^{3}$ Stem Cell Biology and Embryology, Department \\ of Development and Regeneration, KU Leuven, Leuven, Belgium; ${ }^{4}$ Translational Cardiomyology Laboratory, Department of \\ Development and Regeneration, KU Leuven, Leuven, Belgium; ${ }^{5}$ Department of Pharmaceutical and Pharmacological Sciences, KU \\ Leuven, Leuven, Belgium; and ${ }^{6}$ Laboratory of Histology, Morphology Research Group, Biomedical Research Institute, Universiteit \\ Hasselt, Diepenbeek, Belgium
}

Molecular imaging is indispensable for determining the fate and persistence of engrafted stem cells. Standard strategies for transgene induction involve the use of viral vectors prone to silencing and insertional mutagenesis or the use of nonhuman genes. Methods: We used zinc finger nucleases to induce stable expression of human imaging reporter genes into the safe-harbor locus adenoassociated virus integration site 1 in human embryonic stem cells. Plasmids were generated carrying reporter genes for fluorescence, bioluminescence imaging, and human PET reporter genes. Results: In vitro assays confirmed their functionality, and embryonic stem cells retained differentiation capacity. Teratoma formation assays were performed, and tumors were imaged over time with PET and bioluminescence imaging. Conclusion: This study demonstrates the application of genome editing for targeted integration of human imaging reporter genes in human embryonic stem cells for longterm molecular imaging.

Key Words: genome editing; stem cells; reporter genes; PET; noninvasive imaging

J Nucl Med 2017; 58:1659-1665

DOI: 10.2967/jnumed.117.189779

\section{$\mathbf{I}_{\mathrm{n}}$} noninvasive imaging of grafted cells is indispensable for determining their fate and viability. Therefore, noninvasive imaging reporter genes are a suitable tool for long-term tracking of grafted stem cells (1).

Previous research has attempted to deliver exogenous genes into the genome of embryonic stem cells (ESCs) and induced

\footnotetext{
Received Jan. 11, 2017; revision accepted May 1, 2017.

For correspondence or reprints contact: Christophe M. Deroose, UZ Leuven, Division of Nuclear Medicine, Campus Gasthuisberg, Herestraat 49, B-3000 Leuven, Belgium

E-mail: christophe.deroose@uzleuven.be

${ }^{*}$ Contributed equally to this work.

tContributed equally to this work.

Published online Jun. 8, 2017.

COPYRIGHT (C 2017 by the Society of Nuclear Medicine and Molecular Imaging.
}

pluripotent stem cells using various strategies (2). However, longterm follow-up of grafted cells is difficult because of signal silencing $(3,4)$. Furthermore, random and multiple integrations in the host cell genome can perturb the biologic properties and stability of cells, resulting in a nonisogenic cell population (5).

Genome-editing approaches have recently been developed for an efficient targeting approach without random or multiple integrations within the genome of the host cells (6). One example is the use of zinc finger nucleases (ZFNs) (7-10), which contain specific recognition sites of up to 36 base pairs (bp) coupled to a FokI endonuclease domain, generating DNA double-strand breaks on dimerization. These breaks are repaired by either nonhomologous end joining or homologous recombination if a donor plasmid carrying the genes of interest is flanked by sequences homologous to the regions next to the double-strand break. The sequence of interest is then introduced into the host cell genome $(5,7)$.

ZFNs have been designed and used to target the adeno-associated virus integration site 1 ( $A A V S 1$ ) locus, encoding the ubiquitously expressed protein phosphatase 1 regulatory subunit $12 \mathrm{C}$ (PPP1R12C) gene located on chromosome $19(11,12)$. The AAVS1 locus is a safeharbor locus, as the integration of target genes in this locus does not evoke pathologic responses (13) or perturb the proliferation, karyotype, or expression of pluripotency genes in ESCs and induced pluripotent stem cells $(7,14)$. In addition, when ESCs are differentiated, the inserted gene is not silenced.

Here, the AAVS1 locus of ESCs was used to introduce a construct carrying reporter genes for noninvasive imaging. Enhanced green fluorescent protein $(e G F P)$ was introduced for histologic validation. Firefly luciferase (Fluc) was included for bioluminescence imaging (BLI) (15). Finally, a radionuclide imaging reporter gene was included in the construct: either the human sodium iodide symporter (hNIS) or the human somatostatin receptor subtype 2 (hSSTr2). Because the insertion site lies downstream of exon 1 of a transcribed gene, a promoterless puromycin resistance cassette was included in the donor construct to maximize the selection efficiency of correctly targeted cells (14).

The paradigm reporter gene for PET is the herpes simplex virus type 1 thymidine kinase (HSV1-TK) (16-19). This reporter gene has allowed noninvasive documentation in animal models of in vivo gene transfer, cell monitoring, and protein-protein interactions (20) and 
TABLE 1

Primers Used for PCR Genotyping

\begin{tabular}{|c|c|}
\hline PCR reaction & Primer sequences \\
\hline \multirow[t]{2}{*}{$5^{\prime}$ junction assay } & CACTTTGAGCTCTACTGGCTTC \\
\hline & CATGTTAGAAGACTTCCTCTGC \\
\hline \multirow[t]{2}{*}{$3^{\prime}$ junction assay } & TTCACTGCATTCTAGTTGTGG \\
\hline & AAGGCAGCCTGGTAGACA \\
\hline \multirow[t]{2}{*}{$5^{\prime}$ random integration } & GTACTTTGGGGTTGTCCAG \\
\hline & TTGTAAAACGACGGCCAG \\
\hline \multirow[t]{2}{*}{$3^{\prime}$ random integration } & СCTGAGTTCTAACTITGGCTC \\
\hline & ACACAGGAAACAGCTATGAC \\
\hline \multirow[t]{2}{*}{ WT allele } & TTCGGGTCACCTCTCACTCC \\
\hline & GGCTCCATCGTAAGCAAACC \\
\hline
\end{tabular}

has also been used in clinical applications (17-19). Its gene product might be immunogenic; furthermore, if treatment with antiviral drugs is necessary, the HSV1-TK-mediated conversion of the prodrug to its cytotoxic form causes unwanted cell death. Finally, substrates for HSV1-TK might require nucleoside transporters to incorporate the PET probe in the cells, and alterations in transporter status could influence the PET signal.

$\mathrm{h} N I S$ is commonly used for SPECT, PET, and radionuclide therapy of the thyroid gland because of its ability to transport radioactive forms of iodine and other negatively charged ions into thyroid cells $(21,22) . \mathrm{h} N I S$ is principally present in the thyroid. Other tissues, such as the stomach mucosa, mammary glands, or salivary glands, also express hNIS at lower levels (22). This lower uptake implies a low background signal for radionuclide imaging and partly contributes to the interest in the application of $\mathrm{h} N I S$ as a PET reporter gene (23).

hSSTr2 has been imaged for years in the clinic for detection of neuroendocrine tumors using ${ }^{111} \mathrm{In}$-pentetreotide or ${ }^{68} \mathrm{Ga}$-labeled peptides such as ${ }^{68} \mathrm{Ga}$-DOTATATE (24). These molecules are commercially available or are in widespread use for neuroendocrine tumor imaging (25). This receptor is predominantly expressed in the gastrointestinal tract (including the pancreas), the spleen, and the pituitary gland $(24,26)$.

The goal of this study was to generate a stably expressing ESC line for noninvasive, longitudinal multimodality imaging with human PET reporter genes through genome editing with a ZFN-mediated approach.

\section{MATERIALS AND METHODS}

\section{Cell Culture}

The human ESC line H9 (WA09) was purchased from WiCell Research Institute. ESCs were maintained feeder-free using mTeSR1 medium (StemCell Technologies).

\section{Generation of Reporter Cell Lines}

The pZ:puro-CAGGS-eGFP-P2A-Fluc-T2A-hNIS-plasmid and the pZ:puro-CAGGS-eGFP-P2A-Fluc-T2A-hSSTr2-plasmid were generated using the $\mathrm{pZ}$-donor $A A V S 1$ vector (CompoZr Targeted Integration Kit; Sigma Aldrich).

ESCs were resuspended in nucleofection solution 2 (Amaxa; Lonza) with $10 \mu \mathrm{g}$ of donor plasmid and $3 \mu \mathrm{g}$ of ZFN messenger RNA per $2 \times 10^{6}$ cells. The cells were electroporated using program F16. After a week of puromycin selection, individual clones were expanded, resulting in 2 reporter cell lines: $h N I S^{+}$and $h S S T r 2^{+}$ESCs.

\section{Polymerase Chain Reaction (PCR) Genotyping}

Genomic DNA was extracted using the QIAamp DNA mini kit (Qiagen) following the manufacturer's protocol.

To check for reporter gene incorporation, junction assays were performed at the $5^{\prime}$ and $3^{\prime}$ end of the target locus. Random integrations were checked using primers at $5^{\prime}$ and $3^{\prime}$ sites next to the cassette. The wild-type (WT) allele was amplified with primers at the sites adjacent to the insertion site to determine whether the integration was mono- or biallelic. Primer sequences can be found in Table 1 .

\section{Southern Blotting}

Genomic DNA was digested with EcoRI and loaded onto a $0.7 \%$ agarose gel. Fragments were transferred to a nylon membrane (ZetaProbe; Biorad) that was ultraviolet-crosslinked, and prehybridization was performed. Digoxigenin (DIG; Roche) labeling of the probe targeting the homology arm was done by PCR using the donor vector. Hybridization of the membrane with the probe was performed using the DIG High Prime DNA Labeling and Detection Starter Kit II (Roche) according to manufacturer's instructions.

\section{Hepatic Differentiation} (27).

Hepatic differentiation of ESCs was performed as previously described

\section{In Vitro Validation of Reporter Gene Expression}

In vitro BLI was performed as previously described (28).

ESCs were plated, washed, and incubated with tracer solution $(0.74$ $\mathrm{MBq} / \mathrm{mL}$ ) for $1 \mathrm{~h} .{ }^{99} \mathrm{TcO}_{4}{ }^{-}$and iodide $\left({ }^{124} \mathrm{I}^{-}\right)$(Perkin Elmer) were used

TABLE 2

Primers Used for Real-Time Quantitative PCR

\begin{tabular}{lll}
\hline \multicolumn{1}{c}{ Gene } & \multicolumn{1}{c}{ Forward } & \multicolumn{1}{c}{ Reverse } \\
\hline GAPDH & TCAAGAAGGTGGTGAAGCAGG & ACCAGGAAATGAGCTTGACAAA \\
EOMES & AACAACACCCAGATGATAGTC & TCATAGTTGTCTCTGAAGCCT \\
CXCR4 & CACCGCATCTGGAGAACCA & GCCCATTCCTCGGTGTAGTT \\
MIXL1 & GGATCCAGGTATGGTTCCAG & CATGAGTCCAGCTTTAACC \\
SOX17 & CGCTTCATGGTGTGGGCTAAGGACG & TAGTTGGGGTGGTCCTGCATGTGCTG \\
HNF4a & ACTACGGTGCCTCGAGCTGT & GGCACTGGTTCCTCTTGTCT \\
AFP & TGAGCACTGTTGCAGAGGAG & GTGGTCAGTTTGCAGCATTC \\
ALB & ATGCTGAGGCAAAGGATGTC & AGCAGCAGCACGACAGAGTA \\
A1AT & AGGGCCTGAAGCTAGTGGAT & TCCTCGGTGTCCTGACTTC \\
\hline
\end{tabular}




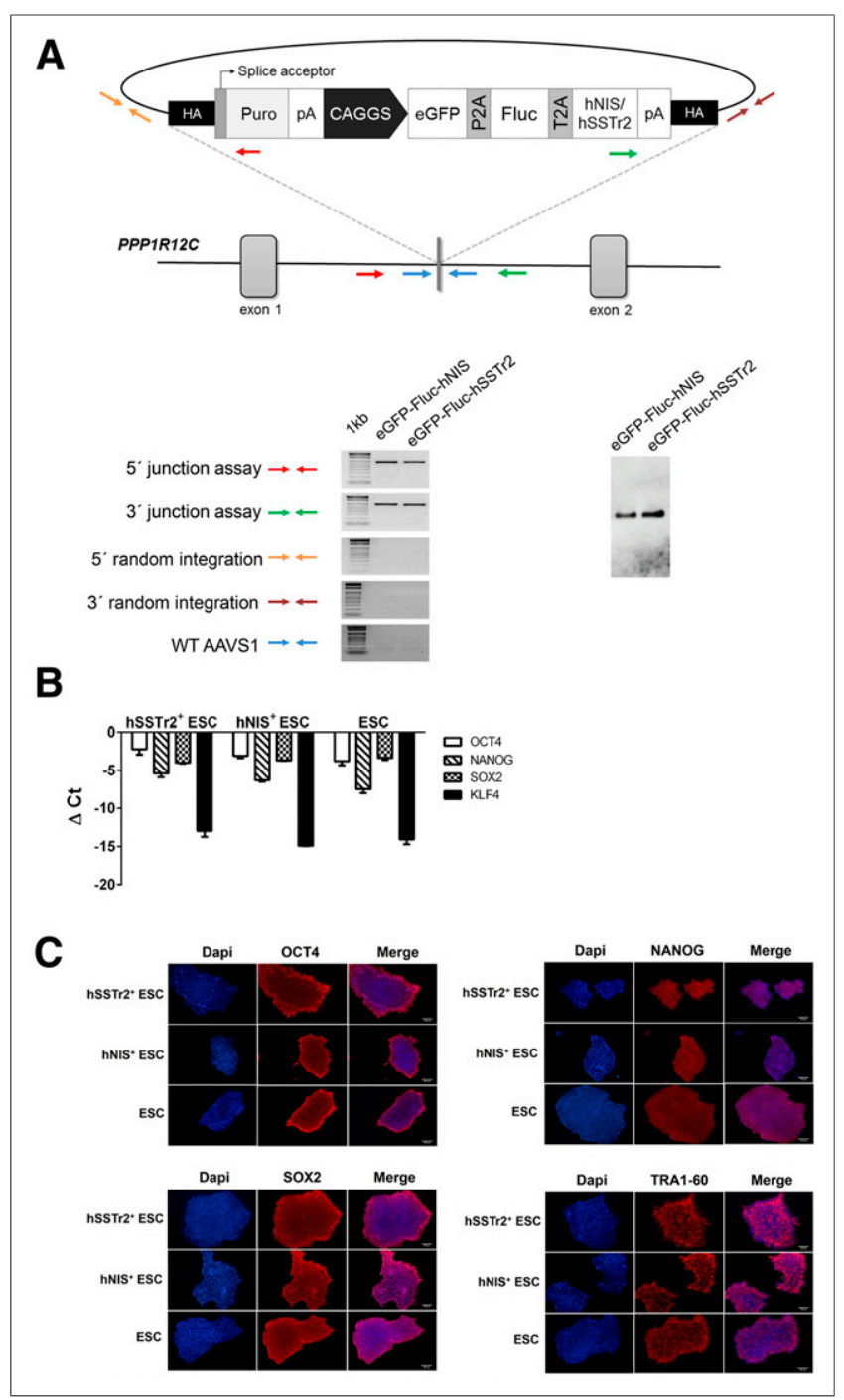

FIGURE 1. Correct integration of transgenes. (A) Recombinant lines incorporating transgenes showed correct integration, and southern blot analysis confirmed correct integration of construct. (B) Real-time quantitative PCR showed no significant difference in expression of pluripotency markers OCT4, NANOG, SOX2, and KLF4 after genetic engineering. (C) Immunocytochemistry for OCT4, NANOG, SOX2, and TRA1-60 showed similar expression in all lines. CAGGS = cytomegalovirus enhancer/chicken $\beta$-actin promoter; $\mathrm{Ct}=$ threshold cycle; Dapi $=4$ ',6-diamidino-2-phenylindole; $\mathrm{HA}=$ homology arms; $\mathrm{pA}=$ polyA tail $\mathrm{P} 2 \mathrm{~A}=$ porcine teschovirus $-12 \mathrm{~A}$; Puro $=$ puromycin resistance gene; $\mathrm{T} 2 \mathrm{~A}=$ thoseaasigna virus $2 \mathrm{~A}$.

for $\mathrm{hNIS}{ }^{+}$ESCs, and ${ }^{68} \mathrm{Ga}$-DOTATATE for $\mathrm{hSSTr}{ }^{+}$ESCs. The cells were washed 3 times, and tracer concentration in the cell fraction was measured using a $\gamma$-counter (Wizard; Perkin Elmer). Values were corrected for cell numbers. $\mathrm{hNIS}$ blocking was performed with $\mathrm{NaClO}_{4}(0.74$ $\mathrm{MBq} / \mathrm{mL}^{99} \mathrm{mcO}_{4}{ }^{-}$in $\left.10 \mu \mathrm{M} \mathrm{NaClO}{ }_{4}\right)$, and $\mathrm{hSSTr} 2$ blocking was performed with cold octreotide $\left(0.74 \mathrm{MBq} / \mathrm{mL}^{68} \mathrm{Ga}\right.$-DOTATATE in $0.08,0.25$, $0.74,2.2,6.7,20$, and $60 \mathrm{nM}$ octreotide). Efflux of ${ }^{99} \mathrm{mcO}_{4}{ }^{-}$was measured by incubating cells with ${ }^{99} \mathrm{TcO}_{4}{ }^{-}$for $1 \mathrm{~h}$, followed by incubation with tracer-free medium for 5, 15, 30, 60, and $120 \mathrm{~min}$. Saturation experiments were performed by incubation of hSSTr $2^{+}$ESCs with different solutions of ${ }^{68} \mathrm{Ga}$-DOTATATE $(0.015,0.022,0.11,0.37$, or $2.22 \mathrm{MBq} / \mathrm{mL})$. Each condition included a blocking with cold octreotide $(20 \mathrm{nM})$. The cells were incubated for $10 \mathrm{~min}$, and activity in the cells was measured after 3 washing steps. On the basis of activity inside the cell and specific activity, the amount of ${ }^{68} \mathrm{Ga}$-DOTATATE bound to the cells was calculated.

\section{Real-Time Quantitative PCR}

Gene expression analysis of pluripotency markers and ESC differentiation toward the hepatic lineage were performed using real-time quantitative PCR as previously described (27). Primer sequences are listed in Table 2.

\section{Immunostaining}

Staining was performed as described previously (28). Goat anti-eGFP (1:500; Abcam), goat anti-Oct4 (1:200; Abcam), rabbit anti-Nanog (1:200; Abcam), goat anti-SOX2 (1:50; Santa Cruz Biotechnology), and mouse anti-TRA1-60 (1:50; Santa Cruz) were used. Antirabbit, antigoat, and antimouse Alexa Fluor 594 (1:500 in 1\% bovine serum albumin; Thermo Fisher) or antigoat Alexa Fluor 488 (1:500; Thermo Fisher) secondary antibodies were used.

\section{Animal Preparation}

All animal protocols were approved by the Ethical Committee of KU Leuven. Eight-week-old female BALB/c Rag2 $2^{-1-} \gamma^{-1-}$ mice were anesthetized with $2 \%$ isoflurane (Isoflurane ISP; Rothacher), and 3-5 $\times 10^{6}$ ESCs were resuspended 1:1 in phosphate-buffered saline and Matrigel (BD Biosciences). hSSTr $2^{+}$ESCs were injected on the left flank, and hNIS ${ }^{+}$ESCs contralaterally $(n=8)$.

\section{BLI}

BLI was performed as previously described (28).

\section{${ }^{68} \mathrm{Ga}$-DOTATATE Preparation}

${ }^{68} \mathrm{Ga}$-DOTATATE was prepared by heating ${ }^{68} \mathrm{Ga}$-chloride $(400-800$ $\mathrm{MBq}$ ) at $\mathrm{pH}$ 4-4.4 with $30 \mu \mathrm{g}$ of DOTATATE (Bachem), according to a previously published method, with adaptation (29). ${ }^{68} \mathrm{Ga}$-chloride was obtained by elution of a ${ }^{68} \mathrm{Ge} /{ }^{68} \mathrm{Ga}$ generator with diluted $\mathrm{HCl}$ solution followed by purification over a Dowex column (SigmaAldrich/Fluka). The reaction mixture was purified over a Sep-Pak C18 column (Waters) and formulated into a clinical-grade injectable solution.

\section{Small-Animal PET}

Imaging was performed on days 1,22 , and 63 after intravenous injection of approximately $3.7 \mathrm{MBq}$ of ${ }^{68} \mathrm{Ga}$-DOTATATE or ${ }^{124} \mathrm{I}^{-}$per mouse $(n \geq 5)$, with static acquisitions of 20 min using a Focus 220 microPET system (Siemens Medical Solutions USA). Time after injection was $1 \mathrm{~h}$ for ${ }^{68} \mathrm{Ga}$-DOTATATE and $2 \mathrm{~h}$ for ${ }^{124} \mathrm{I}^{-}$

Images were reconstructed with a maximum a posteriori algorithm and analyzed using PMOD 3.0 (PMOD Technologies). Images were converted to SUV.

A manually delineated volume of interest was positioned around the teratomas. Ratios were calculated comparing the signal to the contralateral tumor.

\section{Histologic Processing}

Masson trichrome staining was performed using standard protocols and analyzed to identify the presence of derivatives of the 3 germ layers.

\section{Statistical Analysis}

Data are presented as mean \pm SEM. ANOVA was performed. $P$ values of less than 0.05 were considered statistically significant. Data were processed using Prism, version 5.00 (GraphPad Software), for Windows (Microsoft).

\section{RESULTS}

\section{Generation of Reporter Cell Lines}

Two reporter gene constructs were generated, flanked by $800-b p$ stretches of homology to the ZFN target site AAVS1, downstream 


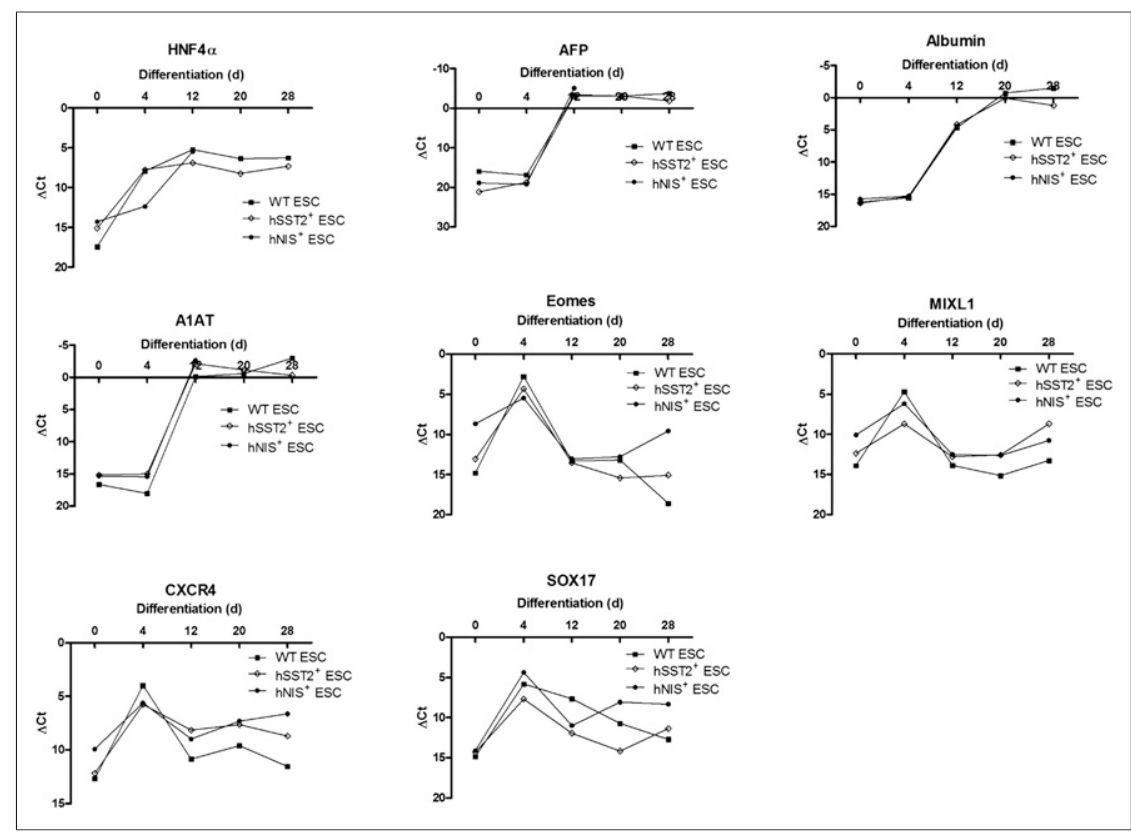

FIGURE 2. No significant difference in hepatic differentiation of $\mathrm{hSSTr}^{+}, \mathrm{hNIS}^{+}$, and WT ESCs. Real-time quantitative PCR for hepatocyte-specific genes HNF4a, AFP, ALB, and A1AT and for definitive endoderm markers EOMES, MIXL1, CXCR4, and SOX17. Ct $=$ threshold cycle.
PCR Genotyping, Southern Blotting, and Expression of Pluripotency Markers

PCR genotyping was performed to assess proper integration of the construct and to exclude random integrations (Fig. 1A). Both $\mathrm{hSSTr}^{+}$and $\mathrm{hNIS}{ }^{+}$colonies carried the expression cassette as confirmed by the $5^{\prime}$ and $3^{\prime}$ junction assays. Single or biallelic integration of the cassette was assessed. In both clones, biallelic integration into the AAVS1 locus was observed. No random integrations had occurred.

Correct integration of the expression construct was confirmed with Southern blotting. A single band was present depicting biallelic integration (Fig. 1A). Expression of the pluripotency markers octamer-binding transcription factor 4 (OCT4), nanog homeobox (NANOG), sex-determining region Y-box 2 (SOX2), and Kruppel-like factor 4 (KLF4) was assessed and found to be the same as for WT ESCs (Fig. 1B). Immunocytochemistry for OCT4, NANOG, SOX2, and TRA1-60 showed a similar expression in all lines (Fig. 1C). of exon 1 of the protein phosphatase 1 regulatory subunit $12 \mathrm{C}$ gene on chromosome 19 (Fig. 1A). The reporter genes were $e G F P$ and Fluc, allowing BLI, and either hNIS or hSSTr2 for PET or SPECT.

\section{Hepatic Differentiation}

WT, hSSTr $2^{+}$, and $\mathrm{hNIS}{ }^{+}$ESCs showed a similar differentiation capacity toward the hepatic lineage (Fig. 2). Expression of the hepatocyte-specific genes hepatocyte nuclear factor $4 \alpha(H N F 4 \alpha)$, $\alpha$-fetoprotein $(A F P)$, albumin $(A L B)$, and $\alpha-1$ antitrypsin $(A 1 A T)$ increased in both cell lines. No significant difference was observed between the WT and the genome-edited ESCs. The definitive endoderm markers eomesdermin (EOMES), MIX1 homeobox-like protein 1 (MIXL1), CXCR4, and SOX17 were expressed similarly in all cell lines.

\section{In Vitro Validation of Reporter Gene Expression}

Both before and after differentiation, genome-edited cells had significantly higher BLI signals than WT ESCs (Fig. 3A; $P<0.05)$.

The functionality of hSSTr 2 and $\mathrm{h} N I S$ was tested with radioligand uptake experiments.

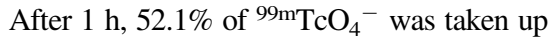
per $5 \times 10^{5} \mathrm{hNIS}{ }^{+}$ESCs, 50-80 times more than in controls $(P=0.001)$. $\mathrm{NaClO}_{4}$ reduced uptake levels significantly $(0.77 \%)$. $\mathrm{hNIS} \mathrm{S}^{+}$hepatocytes maintained an uptake of $40.7 \%$, not significantly different from undifferentiated cells $(52.1 \% ; P>0.05)$ (Fig. 3B). A decreased tracer retention was observed, although after $1 \mathrm{~h}$ about $20 \%$ of the tracer remained intracellular (Fig. 3C).

hSSTr2 ${ }^{+}$ESCs could bind $3.18 \%$ per million cells of the ${ }^{68} \mathrm{Ga}$-DOTATATE, 6.9 times more than $\mathrm{h} N I S^{+}$cells $(P<0.001)$. hSSTr $2^{+}$ hepatocytes maintained their ${ }^{68} \mathrm{Ga}$-DOTATATE 

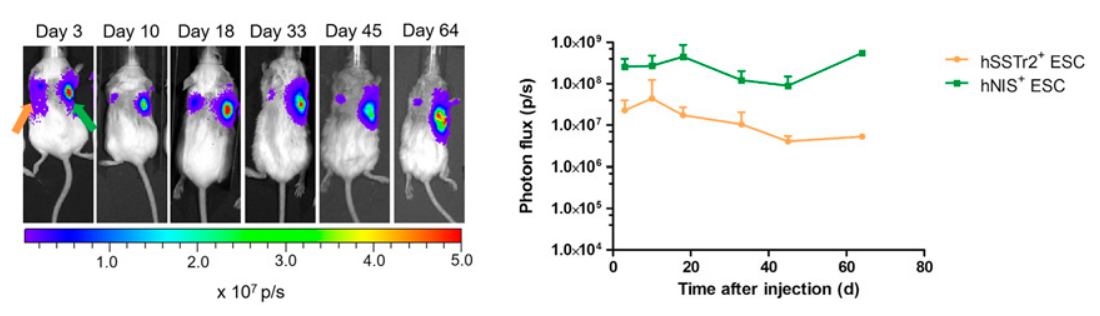

FIGURE 4. BLI of teratomas showed clear signal coming from both $\mathrm{hSSTr} 2^{+}$and $\mathrm{hNIS}{ }^{+} \mathrm{ESCs}$ and persisting over $70 \mathrm{~d}$.

binding capacity. Octreotide administration caused a 4-fold reduction in ${ }^{68} \mathrm{Ga}$-DOTATATE uptake $(0.77 \% ; P<0.001)$ (Fig. 3D). A half-maximal inhibitory concentration of $0.58 \mathrm{nM}$ was demonstrated (Fig. 3E). A saturation experiment showed maximum specific binding of $111 \pm 5 \mathrm{fmol}$ per million cells and a dissociation constant of $3.4 \pm 0.5 \mathrm{nM}$ (Fig. 3F).

\section{In Vivo Noninvasive Longitudinal Imaging}

BLI of hSSTr2 ${ }^{+}$and $\mathrm{hNIS}{ }^{+}$teratomas showed a robust signal that increased over $70 \mathrm{~d}$ (Fig. 4). Small-animal PET was performed on days 1, 22, and 63 (Fig. 5). A focus of increased ${ }^{68} \mathrm{Ga}$-DOTATATE accumulation was observed at the hSSTr $2^{+}$teratoma. No increased ${ }^{68} \mathrm{Ga}$-DOTATATE uptake was seen at the site of the $\mathrm{h} N I S^{+}$teratoma. On day 1 , the hSSTr $2^{+}$ESCs showed $3.07 \pm 0.84$ times more signal than the $\mathrm{hNIS}{ }^{+}$ESCs $(P<0.01)$. This signal increased significantly to $5.02 \pm 2.0$ times greater in the hSSTr2 ${ }^{+}$ESCs on day 63 (Fig. $5 \mathrm{~A}$ ).

On the first day after engraftment of the $\mathrm{hNIS} \mathrm{S}^{+} \mathrm{ESCs}$, a focus of increased tracer uptake was observed, with $2.87 \pm 1.0$ times more accumulation of ${ }^{124} \mathrm{I}^{-}$than in the hSSTr2 ${ }^{+}$teratomas $(P<0.001)$. On day 63 after engraftment, the signal increased significantly to $4.66 \pm 0.7$ times more tracer uptake in $\mathrm{hNIS}{ }^{+}$teratomas than in the controls $(P<0.05)$ (Fig. 5B).

\section{DISCUSSION}

\section{Teratoma Assay}

Histologic examination confirmed teratoma formation and thus maintenance of pluripotency after ZFN targeting. Teratomas from both lines contained cells derived from the 3 different germ layers (Supplemental Fig. 1; supplemental materials are available at http://jnm.snmjournals.org). hSSTr $2^{+}$teratomas formed neural rosettes, cartilage, and intestinal lining epithelium. hNIS ${ }^{+}$teratomas were less dense and more fluid overall, containing neural rosettes, cartilage, and glandular tissue. Immunohistochemistry confirmed expression of hSSTr 2 and $\mathrm{hNIS}$ in $\mathrm{hSSTr} 2^{+}$and $\mathrm{hNIS}{ }^{+}$ teratomas, respectively.

The delivery of genetic material into cells has mainly been done through the use of viral vectors. Hence, insertional mutagenesis may occur, resulting in the disruption of the host genetic material, and there is a potential risk for oncogenesis. Furthermore, nonisogenic cell lines are generated. The primary transduced cell population is a heterogeneous mix of cells that are all genomically different from one another. Furthermore, reporter gene silencing is dependent on the integration site. These factors can result in signal loss and confound data interpretation $(30,31)$.

In this work, we used the innovative ZFN-mediated approach for genetic engineering of ESCs. Human PET imaging reporter genes were introduced into the AAVS1 locus, which is known to be a socalled safe-harbor locus $(7,13,14)$. AAVS1 has a continuously open chromatin structure with insulator elements that prevent epigenetic silencing and thereby allow stable transgene expression, at least when a transgene is expressed from a constitutive active promoter $(7,14)$.

This technology is very translational, as two major limitations of stem cell imaging are tackled at once. First, ZFNs provide safe and controlled introduction of genetic material. Second, human PET reporter genes do not evoke immune responses. The tracers required for these genes are available in routine clinical use and do not require an on-site cyclotron. We used two reporter constructs for multimodal imaging, enabling the monitoring of cells after injection using BLI and PET or SPECT.

In this study, we showed the efficient introduction of the reporter cassette in both alleles of the AAVS1 locus. Very high in vitro uptake ratios were reached $(\sim 2$ orders of magnitude higher than in WT controls), and in vivo imaging was possible when the xenograft was not yet detectable by clinical examination.

$\mathrm{h} N I S$ is a symporter protein and thus mediates an amplification of the imaging signal (22,32). After their uptake through $\mathrm{h} N I S$, no organification of tracer molecules occurs, which leads to partial leakage (28). Nevertheless, hNIS has many advantages such as its human origin, its low background expression, and the availability of
FIGURE 5. PET confirmed tracer uptake at correct locations. (A) ${ }^{68} \mathrm{Ga}$-DOTATATE accumulation observed at hSSTr2 ${ }^{+}$ESC site persisted over time (orange arrows); no signal was present at contralateral $\mathrm{hNIS}{ }^{+}$ESC graft (green arrows). (B) Uptake of ${ }^{124 \mathrm{I}^{-}}$at $\mathrm{hNIS}{ }^{+} \mathrm{ESC}$ site was clear and persisted over time (orange arrows); no signal was present at contralateral hSSTr2 ${ }^{+}$ESC graft (green arrows). 
several tracer molecules that are compatible. As imaging reporter proteins, receptors such as hSSTr2 bind a single ligand in a 1:1 ratio with high affinity (33). No signal amplification or tracer leakage will occur, but hSSTr2 is of human origin and is characterized by low endogenous expression outside some specific endocrine organs.

Both imaging reporter genes have their disadvantages, but their evident advantages make them very good candidates for imaging reporter genes. The choice of the reporter gene will be based mainly on the anatomic location to be imaged, depending on their endogenous expression.

BLI and small-animal PET were performed on $\mathrm{hSSTr}^{+}$and $\mathrm{hNIS}{ }^{+}$ ESCs injected subcutaneously. BLI showed robust signal intensities that further increased specifically. PET signals increased significantly over time and more predominantly in $\mathrm{hSSTr} 2^{+}$ESC teratomas. $\mathrm{hNIS}{ }^{+}$ teratomas contained more fluid-containing cysts not contributing to the signal. $\mathrm{hSSTr}{ }^{+}$teratomas were denser, causing a relatively higher PET signal.

With ${ }^{68} \mathrm{Ga}$-DOTATATE PET, $\mathrm{SUV}_{\text {mean }}$ was $0.07 \pm 0.03$ in $\mathrm{hSSTr}^{+}$and $0.03 \pm 0.02$ in $\mathrm{hNIS}{ }^{+}$teratomas (Fig. 5A). With ${ }^{124} \mathrm{I}^{-} \mathrm{PET}, \mathrm{SUV}_{\text {mean }}$ was $0.21 \pm 0.08 \mathrm{in} \mathrm{h} N I S^{+}$and $0.06 \pm 0.01$ in $\mathrm{SSTr}^{+}$teratomas (Fig. 5B). Imaging of metastasis in a mouse model using melanoma cells expressing HSV1-TK imaged with 9(4-18 F-fluoro-3-hydroxymethylbutyl)guanine $\left({ }^{18} \mathrm{~F}-\mathrm{FHBG}\right)$ resulted in a mean uptake of 3.3 percentage injected dose $(\% \mathrm{ID}) / \mathrm{g}(\mathrm{SUV}, \sim 0.9$; formula from Phelps (34)). Hepatoma and rat glioma cells expressing $H S V 1-T K$ reporter led to an approximately $1.6 \% \mathrm{ID} / \mathrm{g}$ and an approximately $0.7 \% \mathrm{ID} / \mathrm{g} 1-2 \mathrm{wk}$ after engraftment with ${ }^{14} \mathrm{C}$-labeled FIAU ( 2 '-fluoro- 2 '-deoxy- $\beta$-D-arabinofuranosyl-5-iodouracil) and ${ }^{18} \mathrm{~F}-\mathrm{FHBG}$ (35), implying approximate SUVs of 0.32 and 0.14 . Prostate cancer cells expressing HSV1-TK resulted in an uptake of $0.2 \% \mathrm{ID} / \mathrm{g}$, or an SUV of 0.04 (36). Our data suggest slightly lower SUVs; however, others have used transfection or transduction to insert the reporter genes. Hence, multiple copies are inserted, leading to higher expression levels. In our work, a single copy of the reporter gene is included in the genome, and a lower expression level is inevitable. Also, in $\mathrm{hSSTr} 2^{+}$teratomas, lower SUVs were obtained for similar reasons. Furthermore, lower receptor densities were observed than in a previous report (37). To circumvent this problem, antagonists can be used, as they are able to bind more hSSTr2 sites $(37,38)$.

Histologic examination confirmed that ZFN targeting does not alter the pluripotency of the ESCs. Furthermore, in vitro hepatic differentiation was as efficient for hSSTr ${ }^{+}$and $h N I S^{+}$ESCs as for WT ESCs.

Our results are in line with those of Wang et al., who introduced Fluc, monomeric red fluorescent protein, and HSVI-TK in the AAVS1 locus of ESCs and induced pluripotent stem cells (39). A high efficiency of the targeting procedure was shown, with preservation of pluripotency, differentiation capacity, and long-term gene expression. The survival of the cells was monitored with BLI but not with PET. Also, the gene product of HSV-TK is foreign to the human species, implying potential immunologic consequences and unwanted cell death.

Off-target effects have been described $(40,41)$, but the search for novel strategies has led to the discovery of the CRISPR/Cas (clustered regulatory interspaced short palindromic repeats) system (42).

\section{CONCLUSION}

We demonstrated the introduction of human radionuclide reporter genes into the genome of human ESCs in a safe and controlled manner using the innovative ZFN-mediated strategy, thereby bypassing possible immune reactions against the imaging reporter genes.
Furthermore, we performed long-term imaging of ZFN-engineered ESCs using reporter gene small-animal PET. Isogenic cell lines were generated, and these cells could be fully characterized for patient applications. It is clear that genetic engineering in the field of stem cell imaging can greatly accelerate the transition of basic research to a clinical setting, and these innovative techniques can therefore be used to explore other human PET reporter genes.

\section{DISCLOSURE}

Christophe M. Deroose and Koen Van Laere are senior clinical investigators of the Flemish Fund for Scientific Research (FWO). Cindy Casteels is a postdoctoral fellow of FWO. Bryan Holvoet and Natacha Breuls are PhD students funded by IWT. Laura Ordovas was funded by IWT (IWT/OZM/090838), IACS (BPAMER3/08/04), and the Government of Aragon (FMI048/08). Funding to Christophe M. Deroose was from FWO (G.0667.07 and G.0975.11), KU Leuven (ETHC1900-PF and EME-C2161-GOA/11/012), IWT (IWT-HEPSTEM and IWT-HILIM-3D), BELSPO (BELSPO-IUAP-DEVREPAIR), and HeMiBio (FP7-HEALTH, project 266777). No other potential conflict of interest relevant to this article was reported.

\section{ACKNOWLEDGMENTS}

We thank Manja Muijtjens, Pieter Berckmans, Jeanine Santermans, and Ann Van Santvoort for their help in data acquisition and processing. The radiopharmacy staff at Nuclear Medicine UZ Leuven is acknowledged for preparing the ${ }^{68} \mathrm{Ga}$-DOTATATE.

\section{REFERENCES}

1. Yaghoubi SS, Campbell DO, Radu CG, Czernin J. Positron emission tomography reporter genes and reporter probes: gene and cell therapy applications. Theranostics. 2012;2:374-391.

2. Giudice A, Trounson A. Genetic modification of human embryonic stem cells for derivation of target cells. Cell Stem Cell. 2008;2:422-433.

3. Herbst F, Ball CR, Tuorto F, et al. Extensive methylation of promoter sequences silences lentiviral transgene expression during stem cell differentiation in vivo. Mol Ther. 2012;20:1014-1021.

4. Krishnan M, Park JM, Cao F, et al. Effects of epigenetic modulation on reporter gene expression: implications for stem cell imaging. FASEB J. 2006;20:106-108.

5. Collin J, Lako M. Concise review: putting a finger on stem cell biology: zinc finger nuclease-driven targeted genetic editing in human pluripotent stem cells. Stem Cells. 2011;29:1021-1033.

6. Li M, Suzuki K, Kim NY, Liu GH, Izpisua Belmonte JC. A cut above the rest: targeted genome editing technologies in human pluripotent stem cells. J Biol Chem. 2014;289:4594-4599.

7. Hockemeyer D, Soldner F, Beard C, et al. Efficient targeting of expressed and silent genes in human ESCs and iPSCs using zinc-finger nucleases. Nat Biotechnol. 2009;27:851-857.

8. Lombardo A, Genovese P, Beausejour CM, et al. Gene editing in human stem cells using zinc finger nucleases and integrase-defective lentiviral vector delivery. Nat Biotechnol. 2007;25:1298-1306.

9. Zou J, Maeder ML, Mali P, et al. Gene targeting of a disease-related gene in human induced pluripotent stem and embryonic stem cells. Cell Stem Cell. 2009;5:97-110.

10. Raitano S, Ordovas L, De Muynck L, et al. Restoration of progranulin expression rescues cortical neuron generation in an induced pluripotent stem cell model of frontotemporal dementia. Stem Cell Reports. 2015;4:16-24.

11. Kotin RM, Linden RM, Berns KI. Characterization of a preferred site on human chromosome $19 \mathrm{q}$ for integration of adeno-associated virus DNA by non-homologous recombination. EMBO J. 1992;11:5071-5078.

12. Tan I, Ng CH, Lim L, Leung T. Phosphorylation of a novel myosin binding subunit of protein phosphatase 1 reveals a conserved mechanism in the regulation of actin cytoskeleton. J Biol Chem. 2001;276:21209-21216.

13. Smith JR, Maguire S, Davis LA, et al. Robust, persistent transgene expression in human embryonic stem cells is achieved with AAVS1-targeted integration. Stem Cells. 2008;26:496-504. 
14. DeKelver RC, Choi VM, Moehle EA, et al. Functional genomics, proteomics, and regulatory DNA analysis in isogenic settings using zinc finger nucleasedriven transgenesis into a safe harbor locus in the human genome. Genome Res. 2010;20:1133-1142.

15. Keyaerts M, Caveliers V, Lahoutte T. Bioluminescence imaging: looking beyond the light. Trends Mol Med. 2012;18:164-172.

16. Gambhir SS, Bauer E, Black ME, et al. A mutant herpes simplex virus type 1 thymidine kinase reporter gene shows improved sensitivity for imaging reporter gene expression with positron emission tomography. Proc Natl Acad Sci USA. 2000;97:2785-2790.

17. Jacobs A, Voges J, Reszka R, et al. Positron-emission tomography of vectormediated gene expression in gene therapy for gliomas. Lancet. 2001;358:727729.

18. Peñuelas I, Mazzolini G, Boan JF, et al. Positron emission tomography imaging of adenoviral-mediated transgene expression in liver cancer patients. Gastroenterology. 2005;128:1787-1795.

19. Yaghoubi SS, Jensen MC, Satyamurthy N, et al. Noninvasive detection of therapeutic cytolytic T cells with ${ }^{18} \mathrm{~F}$-FHBG PET in a patient with glioma. Nat Clin Pract Oncol. 2009;6:53-58.

20. Paulmurugan R, Massoud TF, Huang J, Gambhir SS. Molecular imaging of drugmodulated protein-protein interactions in living subjects. Cancer Res. 2004;64: 2113-2119.

21. Van Sande J, Massart C, Beauwens R, et al. Anion selectivity by the sodium iodide symporter. Endocrinology. 2003;144:247-252.

22. Dohán O, De la Vieja A, Paroder V, et al. The sodium/iodide symporter (NIS): characterization, regulation, and medical significance. Endocr Rev. 2003;24:4877.

23. Penheiter AR, Russell SJ, Carlson SK. The sodium iodide symporter (NIS) as an imaging reporter for gene, viral, and cell-based therapies. Curr Gene Ther. 2012;12:33-47.

24. Deroose CM, Hindie E, Kebebew E, et al. Molecular imaging of gastroenteropancreatic neuroendocrine tumors: current status and future directions. $\mathrm{J} \mathrm{Nucl}$ Med. 2016;57:1949-1956.

25. Serganova I, Ponomarev V, Blasberg R. Human reporter genes: potential use in clinical studies. Nucl Med Biol. 2007;34:791-807.

26. Reubi JC, Kvols L, Krenning E, Lamberts SW. Distribution of somatostatin receptors in normal and tumor tissue. Metabolism. 1990;39:78-81.

27. Roelandt P, Vanhove J, Verfaillie C. Directed differentiation of pluripotent stem cells to functional hepatocytes. Methods Mol Biol. 2013;997:141-147.
28. Wolfs E, Holvoet B, Gijsbers R, et al. Optimization of multimodal imaging of mesenchymal stem cells using the human sodium iodide symporter for PET and Cerenkov luminescence imaging. PLoS One. 2014;9:e94833.

29. Van Binnebeek S, Vanbilloen B, Baete K, et al. Comparison of diagnostic accuracy of ${ }^{111} \mathrm{In}$-pentetreotide SPECT and ${ }^{68} \mathrm{Ga}$-DOTATOC PET/CT: a lesion-bylesion analysis in patients with metastatic neuroendocrine tumours. Eur Radiol. 2016;26:900-909.

30. Ellis J. Silencing and variegation of gammaretrovirus and lentivirus vectors. Hum Gene Ther. 2005;16:1241-1246.

31. Ramachandra CJ, Shahbazi M, Kwang TW, et al. Efficient recombinase-mediated cassette exchange at the AAVS1 locus in human embryonic stem cells using baculoviral vectors. Nucleic Acids Res. 2011;39:e107.

32. Ahn BC. Sodium iodide symporter for nuclear molecular imaging and gene therapy: from bedside to bench and back. Theranostics. 2012;2:392-402.

33. Cotugno G, Aurilio M, Annunziata $P$, et al. Noninvasive repetitive imaging of somatostatin receptor 2 gene transfer with positron emission tomography. Hum Gene Ther. 2011;22:189-196.

34. Phelps ME. PET: Molecular Imaging and Its Biological Applications. New York, NY: Springer; 2012:130-131.

35. Min JJ, Iyer M, Gambhir SS. Comparison of $\left[{ }^{18} \mathrm{~F}\right] \mathrm{FHBG}$ and $\left[{ }^{14} \mathrm{C}\right] \mathrm{FIAU}$ for imaging of HSV1-tk reporter gene expression: adenoviral infection vs stable transfection. Eur J Nucl Med Mol Imaging. 2003;30:1547-1560.

36. Yang H, Berger F, Tran C, Gambhir SS, Sawyers CL. MicroPET imaging of prostate cancer in LNCAP-SR39TK-GFP mouse xenografts. Prostate. 2003;55: 39-47.

37. Reubi JC, Schar JC, Waser B, et al. Affinity profiles for human somatostatin receptor subtypes SST1-SST5 of somatostatin radiotracers selected for scintigraphic and radiotherapeutic use. Eur J Nucl Med. 2000;27:273-282.

38. Reubi JC, Waser B, Macke H, Rivier J. Highly increased ${ }^{125}$ I-JR11 antagonist binding in vitro reveals novel indications for sst 2 targeting in human cancers. J Nucl Med. 2017;58:300-306.

39. Wang Y, Zhang WY, Hu S, et al. Genome editing of human embryonic stem cells and induced pluripotent stem cells with zinc finger nucleases for cellular imaging. Circ Res. 2012;111:1494-1503.

40. Cathomen T, Joung JK. Zinc-finger nucleases: the next generation emerges. Mol Ther. 2008;16:1200-1207.

41. Pattanayak V, Ramirez CL, Joung JK, Liu DR. Revealing off-target cleavage specificities of zinc-finger nucleases by in vitro selection. Nat Methods. 2011;8:765-770.

42. Pennisi E. The CRISPR craze. Science. 2013;341:833-836. 